\title{
FRAMEWORK PARA LA ENSEÑANZA DE LA INVESTIGACIÓN EN LOS POSGRADOS DE EDUCACIÓN
}

\author{
FRAMEWORK PARA O ENSINO DA PESQUISA NAS PÓS-GRADUAÇÕES EM \\ EDUCAÇÃO
}

\author{
FRAMEWORK FOR TEACHING OF RESEARCH IN POSTGRADUATE \\ COURSES IN EDUCATION
}

\author{
Roman Eduardo Sarmiento PORRAS ${ }^{1}$ \\ Lina Maria OSORIO VALDÉS ${ }^{2}$
}

RESUMEN: La autonomía de la cátedra en la Universidad es fundamental para el crecimiento de la academia y la investigación. No obstante, esa singularidad puede convertirse en un obstáculo para el intercambio de estudiantes, homologación de títulos y en general, para la adecuada interacción entre estudiantes-investigadores-docentes de diferentes universidades (dentro y fuera del país). El fortalecimiento de los programas educativos surge fundamentalmente de los procesos de investigación. Hoy, en una sociedad globalizada, cada vez es más común encontrar estudiantes y docentes que buscan intercambios académicos y de investigación con el fin de ampliar su perfil académico y profesional. Sin embargo, son pocos los que logran encontrar Universidades afines a sus actuales procesos de formación y de enseñanza. Este artículo describe de manera detallada, después de dos años de recolección de información cualitativa y cuantitativa, un framework común sobre la enseñanza de la investigación en programas de posgrado en educación. Este framework busca iniciar la discusión, desde las Ciencias Sociales y Humanas, sobre lo valioso y riguroso que debe ser para las universidades el proceso de investigación en sus posgrados. Esto permitirá crear estrategias de intercambio y fortalecimiento, desde la investigación, de los programas académicos.

PALABRAS CLAVE: Investigación en Educación. Framework de investigación. Posgrados e investigación.

RESUMO: A autonomia da cátedra da Universidade é essencial para o crescimento da academia e da pesquisa. No entanto, essa singularidade pode se tornar um obstáculo para o intercâmbio de alunos, homologação de cursos e, em geral, para a interação adequada entre alunos-pesquisadores-professores de diferentes universidades (dentro e fora do pais). $O$ fortalecimento dos programas educacionais decorre fundamentalmente dos processos de pesquisa. Hoje, em uma sociedade globalizada, é cada vez mais comum encontrar alunos e professores em busca de intercâmbios acadêmicos e de pesquisa para ampliar seu perfil

\footnotetext{
${ }^{1}$ Universidad Autónoma de Bucaramanga (UNAB), Bucaramanga - Santander - Colombia. Docente del Departamento de Matemáticas y Ciencias Naturales. Doctor en Tecnología Educativa (SIU) - Illinois. ORCID: https://orcid.org/0000-0002-8647-8659. E-mail: rsarmiento@unab.edu.co

${ }^{2}$ Universidad Autónoma de Bucaramanga (UNAB), Bucaramanga - Santander - Colombia. Coordinadora de Posgrados Facultad de Ciencias Sociales, Humanidades y Artes. ORCID: https://orcid.org/0000-0003-2305-9630. E-mail:losorio3@unab.edu.co
} 
acadêmico e profissional. No entanto, poucos são capazes de encontrar Universidades relacionadas aos seus atuais processos de formação e ensino. Este artigo descreve em detalhes, após dois anos de coleta de informações qualitativas e quantitativas, um framework comum para o ensino da pesquisa em programas de pós-graduação em educação. Este framework busca iniciar a discussão, a partir das Ciências Sociais e Humanas, sobre quão valioso e rigoroso deve ser o processo de pesquisa em seus programas de pós-graduação para as universidades. Isso permitirá a criação de estratégias de intercâmbio e fortalecimento, com base na pesquisa, de programas acadêmicos.

PALAVRAS-CHAVE: Pesquisa em Educação. Framework da pesquisa. Pós-graduação e pesquisa.

ABSTRACT: The autonomy of teaching in the University is fundamental for the growth of academia and research. Nevertheless, this singularity can become an obstacle for students' exchanges, degrees homologation and in general, for the adequate participation among students-researchers-teachers of different universities (inside and outside the country). The strengthening of educational programs arises mainly from the research processes. Today, in a globalized society, it is increasingly common to find students and teachers who seek academic and research exchanges to expand their academic and professional profile. However, there are few who manage to find universities with similar interest and homogeneous and teaching processes. This article describes in detail, after two years of collecting qualitative and quantitative information, a common framework on the teaching of research in graduate programs in education. This framework seeks to open the discussion among academics about how valuable and rigorous the research process in graduate programs should be in the University. This will allow new strategies, to promote research and academic exchange within graduate academic programs.

KEYWORDS: Research in Education. Research framework. Postgraduate and research.

\section{Introducción}

La enseñanza de la investigación se convierte en un componente fundamental dentro de los planes de estudio -y en especial en los posgrados, dado que a través de esta se busca que los estudiantes se acerquen a la realidad, al conocimiento y a la información, más aún cuando estos aspectos son el capital más valioso de las personas, las empresas y en general, la sociedad. Sin embargo, la Educación Superior sigue enfrentándose a la disyuntiva entre formar científicos o formar profesionales, la cual, hoy por hoy, recae en el nivel de posgrados, gracias a su notable crecimiento en las últimas décadas (DÁVILA, 2012).

En la medida en que la oferta de posgrado crece, también crecen las dinámicas frente a estos en los diferentes países, de la mano de las políticas públicas, la reglamentaciones educativas nacionales, entre otros, lo cual se ve reflejado en modalidades e intereses diferentes o comunes, formación de recurso humano altamente calificado, uso de Tecnologías de la 
Información y Comunicación (TIC), vinculación del posgrado con la investigación, procesos de evaluación, acreditación de alta calidad e internacionalización (DÁVILA, 2012).

A nivel general, cada país cuenta con una legislación y regulación sobre los posgrados. Sin embargo, uno de los componentes más complejos en la educación, es la investigación. La contradicción de perspectivas y dinámicas al interior de cada programa de posgrados incrementan la dificultad de encontrar nuevo conocimiento, producirlo, evaluarlo y, por ende, aplicarlo. Esto, paradójicamente, contradice la misión, visión y planes de estudios de la mayoría de las universidades dado que allí se describe la investigación como uno de sus principales pilares institucionales de formación.

En Colombia, más del 90\% de los egresados de los programas de pregrado no manejan el proceso investigativo, es decir: no saben formular un proyecto de investigación, ejecutarlo e integrar el conocimiento que recopilan. Por lo tanto, al llegar al posgrado, el estudiante trae una serie de vacíos que, sumados a un número reducido de horas semanales de dedicación, la poca preparación de los docentes y los limitados espacios de trabajo alrededor de la misma, tienen como consecuencia pobres resultados que difícilmente se orientan a la resolución de problemas reales (CARVAJAL, 2000).

Teniendo en cuenta lo anterior, es posible pensar en la necesidad de un framework que articule las formas de enseñar investigación, sus productos y sus verdaderos alcances. Esto permitiría la formación de estudiantes/egresados con capacidades investigativas acordes a las necesidades de la sociedad. La calidad, pertinencia e impacto de las investigaciones producto de estos procesos de formación a nivel de posgrados estarían encaminadas a la internacionalización no solo de sus resultados, sino, de igual forma, de los estudiantes/egresados y, por ende, de los mismos programas académicos. Esto estaría alineado con lo establecido en la Ley Colombiana 30 de 1992, artículos 10, 11, 12 y 13 en relación con el papel que se espera, juegue la investigación en los posgrados, entendida esta, como la búsqueda y generación de conocimiento.

En la actualidad, los estudiantes de posgrado como producto de su actividad investigativa entregan una monografía, tesis o trabajo de grado. Pero, ¿cuáles son los recursos que ofrecen las universidades para acompañar este proceso de construcción de conocimiento?, ¿quiénes son las personas que acompañan ese proceso?, ¿qué debería saber un estudiante de posgrado para llevar a feliz término su ejercicio de investigación?, ¿qué debería enseñarse al formar en investigación?, estas son algunas de las preguntas de investigación que el estudio resolvió y se presentan en este artículo, de tal forma que el framework propuesto permite marcar una guía frente a qué, cómo y quienes deberían enseñar investigación en posgrados en Ciencias 
Sociales y Humanas.

Este ejercicio de investigación es de corte mixto. Si bien se definió una muestra de 69 docentes y 250 estudiantes de posgrados en Ciencias Sociales y Humanas, distribuidos en las 5 universidad participantes en diferentes países de Iberoamérica; este artículo se concentra en los resultados obtenidos en la Universidad Autónoma de Bucaramanga UNAB, y los productos propuestos para el programa de Maestría en Educación, a partir de los mismos.

\section{Objetivos}

La intención inicial de este artículo es compartir con la comunidad académica un framework que soporte los procesos de enseñanza de la investigación en los posgrados de Educación. Para lo anterior, se presentan los resultados que hicieron posible la construcción de este y sus diferentes aplicaciones.

Dentro del marco de la investigación realizada se propuso como objetivo general el diseñar un framework de enseñanza para el planteamiento e implementación de proyectos de investigación en posgrados en Educación. Para el desarrollo de este fue necesario establecer criterios de recolección de información en cuatro momentos:

(1) Diagnosticar los conocimientos y habilidades de ingreso de los estudiantes de posgrados para el diseño e implementación de proyectos de investigación en Educación,

(2) Identificar la formación y experiencia de los docentes que participan en el proceso de enseñanza y acompañamiento de la investigación en posgrados en Educación,

(3) Caracterizar el proceso de enseñanza de la investigación, a través de la revisión de planes de estudio de programas de este nivel en diferentes universidades,

(4) Identificar las temáticas más comúnmente investigadas por los estudiantes de posgrado en Educación.

Si bien es clara la intención inicial del estudio, es importante aclarar, que más allá de completar con éxito sus objetivos y de presentarlos de manera articulada y coherente en este escrito, se busca aportar de manera significativa a la discusión sobre la función de la investigación en los procesos de formación e internacionalización de los posgrados.

\section{Desarrollo}

\section{Metodología}


La metodología utilizada fue mixta dado que para lograr todos los objetivos propuestos se hizo necesario el análisis de instrumentos desde lo cualitativo y cuantitativo. La triangulación (HERNÁNDEZ; FERNÁNDEZ; BAPTISTA, 2000) buscó obtener información a través de la utilización de cuestionarios con preguntas abiertas (a docentes y estudiantes), y análisis de contenido (guías cátedra, planes de área, estructura curricular, documento maestro del programa, entre otros).

Lo anterior permitió establecer: (1) una línea base sobre las percepciones de parte de los docentes sobre lo qué se debe enseñar en investigación, (2) el ideal en términos de enseñanza de la investigación en posgrado, propuesto por las instituciones educativas, (3) los imaginarios, por parte de los estudiantes, sobre lo que debería ser enseñado para llegar a feliz término en su proyecto de investigación y (4) el nivel real de ingreso de los estudiantes y docentes frente a las competencias y contenidos de los módulos de investigación.

Es así, como a partir de la triangulación de estas fuentes, se estableció el verdadero estado de los procesos de investigación en los programas de posgrado que se analizaron. El análisis cuantitativo, de igual manera, se implementó de forma transversal en el desarrollo de cada objetivo propuesto. Inicialmente se realizó un análisis cuantitativo descriptivo, sobre el nivel de conocimiento de los estudiantes y docentes del área de investigación, se crearon perfiles de ingreso de estudiantes haciendo especial énfasis en sus competencias investigativas, y para el caso del docente un ejercicio similar más allá de los títulos académicos que el docente posee.

Este estudio es mixto, dado el diseño que se buscó implementar para la recolección de información, no fue experimental, en la medida en que se estudió, en su estado natural, sin alteraciones o control por parte de los investigadores una situación particular. No existió manipulación de variables de manera intencional, sino, que, por el contrario, los módulos de investigación, los docentes y estudiantes de los programas de posgrado fueron estudiados en su contexto natural. Esto permitió su posterior análisis. Finalmente, se concibe como un estudio transeccional, dado que el alcance final de la investigación fue de tipo descriptivo; para esto se recolectaron datos en un momento único, buscando conocer la manera en que se hace evidente cada una de las variables y categorías que se acotan el documento o que pudieron emerger de mismo estudio (HERNÁNDEZ; FERNÁNDEZ; BAPTISTA, 2010).

Aunque la población objeto de estudio inicial de la investigación fueron los estudiantes que cursaron en el pasado o que se encontraban cursando algún posgrado en Ciencias Sociales y Humanas en las Universidades participantes y que en su plan curricular se encontraban tomando el curso de investigación como parte de su formación en las diferentes universidades 
participantes: Universidad Cristóbal Colón (México), Universidad Estatal Paulista Julio de Mesquita Filho (Brasil), Universidad de Granada (España), Universidad Academia de Guerra de Chile (Chile) y la Universidad Autónoma de Bucaramanga (Colombia); este artículo se concentra en el framework diseñado específicamente para el programa de Maestría en Educación de la Universidad Autónoma de Bucaramanga. La Tabla 1, presenta la descripción de la población y muestra seleccionada y participante en cada universidad.

Tabla 1 - Distribución del universo, muestra esperada y muestra real de participantes

\begin{tabular}{l|c|c|c|c|c|c|c|c|c}
\hline & \multicolumn{3}{|c|}{ TAMAÑO DEL UNIVERSO } & \multicolumn{3}{c|}{ MUESTRA ESPERADA } & \multicolumn{4}{c}{$\begin{array}{c}\text { NÚMERO REAL DE } \\
\text { PARTICIPANTES }\end{array}$} \\
\hline Universidad & Estudiantes & $\begin{array}{c}\text { Graduado } \\
\mathrm{s}\end{array}$ & $\begin{array}{c}\text { Docentes de } \\
\text { Investigación }\end{array}$ & $\begin{array}{c}\text { Estudiante } \\
\mathrm{s}\end{array}$ & $\begin{array}{c}\text { Graduado } \\
\mathrm{s}\end{array}$ & $\begin{array}{c}\text { Docentes de } \\
\text { Investigación }\end{array}$ & $\begin{array}{c}\text { Estudiante } \\
\mathrm{s}\end{array}$ & $\begin{array}{c}\text { Graduado } \\
\mathrm{s}\end{array}$ & $\begin{array}{c}\text { Docentes de } \\
\text { Investigación }\end{array}$ \\
\hline $\begin{array}{l}\text { Académica } \\
\text { de Guerra } \\
\text { (Chile) }\end{array}$ & $\begin{array}{c}11+151= \\
162\end{array}$ & 31 & 2 & 115 & 29 & 2 & 1 & 2 & 1 \\
\hline $\begin{array}{l}\text { UCC } \\
\text { (México) }\end{array}$ & 79 & 34 & 3 & 66 & 32 & 3 & 8 & 9 & 1 \\
\hline $\begin{array}{l}\text { UNAB } \\
\text { (Colombia) }\end{array}$ & 138 & 138 & 6 & 104 & 104 & 6 & 126 & 85 & 6 \\
\hline $\begin{array}{l}\text { Universidad } \\
\text { de Granada } \\
\text { (España) }\end{array}$ & 30 & 21 & 58 & 28 & 20 & 51 & 3 & 14 & 8 \\
\hline
\end{tabular}

Fuente: Elaborado por los autores

Luego de la revisión bibliográfica y de la reflexión teórica sobre el problema, se definieron las actividades y las técnicas e instrumentos usadas de acuerdo con cada uno de los objetivos planteados. Como resultado inicial se lograron establecer los instrumentos a utilizar para la recolección y análisis de la información. Se diseñaron dos formularios: (1) uno que permitiera recolectar la información relacionada con la formación y experiencia profesional del docente del nivel de posgrado del área de ciencias sociales y humanas y (2) un cuestionario para detectar las competencias investigativas de los estudiantes y/o egresados de posgrados.

\section{Formulario para docentes}

Este formulario permitió identificar las características principales alusivas a la formación y experiencia de los docentes que hacían parte del proceso de enseñanza de la investigación en las universidades participantes. Las categorías utilizadas permitieron obtener datos cuantitativos acerca de los perfiles de los docentes que imparten asignaturas/módulos de investigación en los centros educativos involucrados en el proyecto, las categorías que conforman este instrumento son las siguientes: 
- Información general de la universidad: datos referentes al nombre de la universidad, ubicación, tipo de posgrado al que pertenece.

- Datos generales del profesor: información sociodemográficos relacionados con la edad y el sexo. Además de los años de antigüedad en la universidad y el tipo de contratación que tiene.

- Formación académica: Grado académico de cada docente. Lo anterior para validar que quienes enseñan a investigar y desarrollen proyectos que cuenten con formación/especialización en Ciencias Sociales y Humanidades.

- Experiencia profesional: labores que desempeñadas los docentes.

- Experiencia en investigación: información correspondiente a los trabajos de investigación realizados, talleres, asesorías de tesis, funciones como directores de trabajos finales o sinodal en exámenes profesionales. Además de la participación en eventos de difusión y divulgación científica.

- Trabajo colaborativo: información relacionada con los equipos, redes, comités académicos y editoriales de los que forman parte.

- Publicaciones: experiencia en la publicación de artículos en revistas indexadas o del área de Ciencias Sociales y Humanidades.

- Gestión: información sobre las labores de coordinación y participación en la organización de eventos, foros, presentación de libros o proyectos de investigación que hayan llevado a cabo los profesores o apoyado para la difusión y divulgación de resultados de estudios en el campo de las disciplinas en Ciencias Sociales y Humanidades.

\section{Formulario para estudiantes y graduados}

Este instrumento identificó las competencias y percepciones relacionadas con la enseñanza y uso de la investigación por parte de los estudiantes y egresados. Se definieron tres categorías en el instrumento:

- Información General: al igual que en el instrumento de los docentes, se hizo necesario recolectar la información relacionada con la Universidad de origen de los estudiantes y egresados.

- Formación Teórica: información sobre los cursos, módulos o clases de investigación que ha tomado a lo largo de su formación posgradual, los métodos de investigación que reconoce en su ejercicio académico y cómo desde sus conocimientos, reconoce elementos teóricamente fundamentales, en el desarrollo de su proceso de investigación.

- Experiencia en Investigación: identifica cuál es el nivel de práctica de los contenidos que en el aula son enseñados, asimismo, establece los tipos de proyectos y las metodologías que más se aplican al contexto real.

\section{Resultados}

Este apartado se desarrolló intentado dar respuesta a los objetivos propuestos en la investigación, pero con los productos específicamente diseñados para el programa de Maestría en Educación de la Universidad Autónoma de Bucaramanga UNAB.

- Diseñar un framework que soporte los procesos de enseñanza de la investigación en los posgrados de Educación. El mismo se materializó a través de la propuesta de un protocolo de investigación para la Maestría en Educación, que ofrece información sobre el proceso de investigación como tal, así como también sobre los participantes en el mismo: docentes, estudiantes y coordinadores del programa. Igualmente, en este documento se realiza una propuesta frente a los módulos de investigación, que para este caso son cuatro (uno por semestre), en los cuales el 
estudiante además de recibir información teórica sobre el proceso de investigación, irá realizando progresivamente su trabajo final de grado (ver Tabla 2).

- Caracterizar el proceso de formación en investigación en posgrados de educación, a través de la revisión de planes de estudio de programas de este nivel en diferentes universidades. Para desarrollar este objetivo, fueron de gran utilidad las diversas sesiones de trabajo sincrónico (a través de BB Collaborate) y asincrónico (foro en TEMA), con las diversas universidades participantes (UCC; UNAB, UGR, UNESP y Académica de Guerra). Lo anterior permitió compartir información sobre el programa que eligieron para ser revisado en el ejercicio general de investigación. De esta manera se recolectó información de los programas relacionada con: duración total, el perfil profesional de los estudiantes y de los egresados, los módulos de investigación, los semestres en que se imparten y las competencias que se espera el estudiante adquiera. Esta caracterización, posibilitó encontrar puntos en común entre las universidades participantes que sirvieron como base para definir el número de horas y cursos de investigación, así como su metodologías, aprendizaje y productos esperados, para el caso de la UNAB, se propuso un proceso de investigación compuesto por 4 cursos, cada uno con 3 créditos que equivales a 36 horas de trabajo directo en el aula con un docente de investigación, más el trabajo fuera de ella, que se lleva a cabo con el director del trabajo de grado (ver tabla 2).

Tabla 2 - Proceso de investigación Maestría en Educación UNAB.

\begin{tabular}{|c|c|c|c|c|}
\hline SEMESTRE & MÓDULO & ENTREGABLES & $\begin{array}{l}\text { EVALUACIÓN- } \\
\text { RESPONSABLE } \\
\end{array}$ & OBSERVACIONES \\
\hline Primero & $\begin{array}{l}\text { Trabajo de } \\
\text { grado } 1\end{array}$ & $\begin{array}{l}\text { Anteproyecto } \\
\text { Capítulo } 1\end{array}$ & $\begin{array}{l}\text { Ejercicios realizados en las sesiones } \\
\text { del módulo (40\%)- Profesor del } \\
\text { módulo } \\
\text { Anteproyecto (30\%)- Profesor del } \\
\text { módulo } \\
\text { Capítulo 1 (30\%)- Director } \\
\text { asignado }\end{array}$ & $\begin{array}{l}\text { Se tendrán } 3 \text { sesiones de } \\
\text { clase consecutivas. } \\
\text { A partir de la base de datos } \\
\text { de directores, el estudiante } \\
\text { podrá seleccionar dos } \\
\text { posibles profesores para } \\
\text { dirigir su trabajo. Una vez } \\
\text { el Coordinador académico } \\
\text { valide dicha selección la } \\
\text { remitirá al profesor de } \\
\text { trabajo de grado 1, quien se } \\
\text { encargará de establecer } \\
\text { contacto entre el posible } \\
\text { director y el estudiante, } \\
\text { mediante carta de } \\
\text { invitación por correo } \\
\text { electrónico para confirmar } \\
\text { el interés de trabajar juntos. }\end{array}$ \\
\hline Segundo & $\begin{array}{l}\text { Trabajo de } \\
\text { grado } 2\end{array}$ & $\begin{array}{l}\text { Capítulo } 2 \\
\text { Capítulo } 3\end{array}$ & $\begin{array}{l}\text { Ejercicios realizados en las sesiones } \\
\text { del módulo (40\%)- Profesor del } \\
\text { módulo } \\
\text { Capítulo } 2(30 \%) \text { - Director } \\
\text { asignado } \\
\text { Capítulo } 3(30 \%) \text { - Director } \\
\text { asignado }\end{array}$ & $\begin{array}{l}\text { Se tendrán } 4 \text { sesiones de } \\
\text { clase de } 9 \text { horas cada una, } \\
\text { que no serán consecutivas } \\
\text { y se distribuirán a lo largo } \\
\text { del semestre antes del } \\
\text { inicio de cada uno de los } \\
\text { otros módulos. }\end{array}$ \\
\hline Tercero & $\begin{array}{c}\text { Trabajo de } \\
\text { grado } 3\end{array}$ & Capítulo 4 & $\begin{array}{l}\text { Ejercicios realizados en las sesiones } \\
\text { del módulo (40\%)- Profesor del } \\
\text { módulo } \\
\text { Capítulo } 4(60 \%) \text { - Director } \\
\text { asignado }\end{array}$ & $\begin{array}{l}\text { Se tendrán } 4 \text { sesiones de } \\
\text { clase de } 9 \text { horas cada una, } \\
\text { que no serán consecutivas } \\
\text { y se distribuirán a lo largo } \\
\text { del semestre antes del } \\
\text { inicio de cada uno de los } \\
\text { otros módulos. }\end{array}$ \\
\hline
\end{tabular}

RIAEE - Revista Ibero-Americana de Estudos em Educação, Araraquara, v. 15, n. esp. 4, p. 2653-2670, dez., 2020. e-ISSN: 1982-5587 


\begin{tabular}{|c|c|c|c|c|}
\hline Cuarto & $\begin{array}{c}\text { Trabajo de } \\
\text { grado } 4\end{array}$ & $\begin{array}{c}\text { Capítulo } 5 \\
\text { Documento final } \\
\text { Artículo } \\
\text { (modalidad de } \\
\text { investigación) } \\
\text { Sustentación }\end{array}$ & $\begin{array}{l}\text { Documento final y artículo, este } \\
\text { último para la modalidad de } \\
\text { investigación. El director revisa y } \\
\text { da su aval para que el trabajo sea } \\
\text { enviado a lectura de un evaluador. } \\
\text { Si el director no da su aval, el } \\
\text { estudiante debe matricular } \\
\text { nuevamente el módulo Trabajo de } \\
\text { grado } 4 . \\
\text { Si el director da su aval, el } \\
\text { documento final, se envía a lectura } \\
\text { de un evaluador externo quien } \\
\text { revisa el trabajo y emite su } \\
\text { concepto para avalar o no la } \\
\text { presentación de la sustentación. } \\
\text { Sustentación (100\%)- Distribuido } \\
\text { así: } \\
\text { Evaluador (50\%) } \\
\text { Profesor de Trabajo de grado } 4 \\
\text { (50\%) } \\
\text { El director participa en la } \\
\text { sustentación con voz, pero sin voto } \\
\text { en la calificación. } \\
\text { Para aprobar la sustentación se } \\
\text { requiere una calificación mínima de } \\
3.5\end{array}$ & $\begin{array}{l}\text { Se tendrán } 3 \text { sesiones de } \\
\text { clase que no serán } \\
\text { consecutivas y se } \\
\text { distribuirán a lo largo del } \\
\text { semestre antes del inicio de } \\
\text { los otros módulos. } \\
\text { Las horas correspondientes } \\
\text { a la cuarta sesión serán } \\
\text { empleadas en la } \\
\text { sustentación. }\end{array}$ \\
\hline
\end{tabular}

Fuente: Protocolo de investigación. Maestría en Educación UNAB (2018)

- Diagnosticar los conocimientos y habilidades de ingreso de los estudiantes de posgrados para el diseño e implementación de proyectos de investigación. Para lograr dar respuesta a este objetivo se recopiló información de los estudiantes activos y egresados de programas de posgrados en Ciencias Sociales y Humanas. Al final de este ejercicio (enero de 2017- julio de 2018) se habían encuestado un total de 250 estudiantes, entre estudiantes activos $(56 \%)$ y graduados $(44 \%)$ (Ver Figura 1). Estando de esta manera, la mayor concentración de respuestas en Colombia $(84,4 \%)$, España $(7,6 \%)$, México $(6,8 \%)$ y Chile $(1,2 \%)$. De los encuestados $70 \%$ son mujeres, el $30 \%$ hombres (Ver Figura 2), y se encuentran en un rango de edades entre los 30 y 55 años.

Figura 1 - Estatus de los participantes en la encuesta

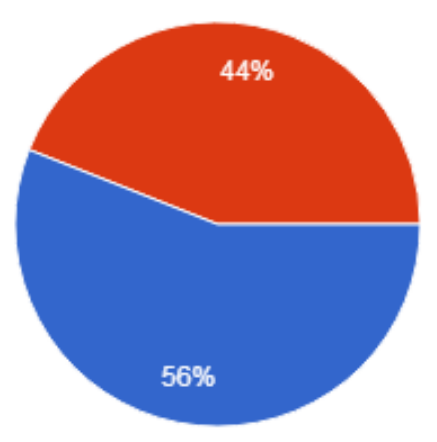

Fuente: Elaborado por los autores 
Figura 2 - Género de los participantes en la encuesta

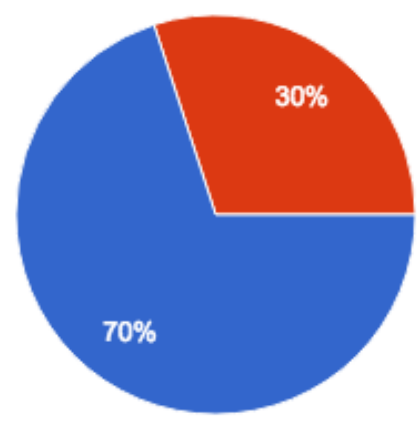

Femenino

Masculino

Fuente: Elaborado por los autores

Frente a la profesión actual de las personas que ingresan a realizar un posgrado en Ciencias Sociales y Humanas, es muy variada. El 69,2\% de las personas encuestadas son docentes (173), pero también es posible encontrar otras profesiones como: sacerdotes, arquitectos, administradores, abogados, ingenieros, músicos, sociólogos, biólogos, comunicadores sociales, periodistas, diseñadores de modas, fisioterapeutas, psicólogos, enfermeras, médicos y trabajadores sociales.

Cuando los estudiantes llegan al nivel de posgrado, en este caso maestría, en su gran mayoría $(73,2 \%)$ traen de su pregrado una formación teórica sobre investigación. Atendiendo a esta respuesta, se les pidió a los encuestados que calificaran su conocimiento en los diferentes métodos de investigación cualitativo, cuantitativo y mixto. Los encuestados indicaron que su conocimiento frente al método cuantitativo es regular $(33,2 \%)$, bueno $(29,6 \%)$, y sólo el $6,4 \%$ lo calificó como muy bueno. Para el caso del método cualitativo, el 43,6\% califica sus conocimientos como bueno, seguido de un $27,6 \%$ que indica que es regular. Finalmente, para el caso del método mixto el $39,2 \%$ lo califica como regular, seguido por un $26,8 \%$ que indica que es bueno (ver Figura 3). 
Figura 3 - Conocimiento frente a los diferentes métodos de investigación

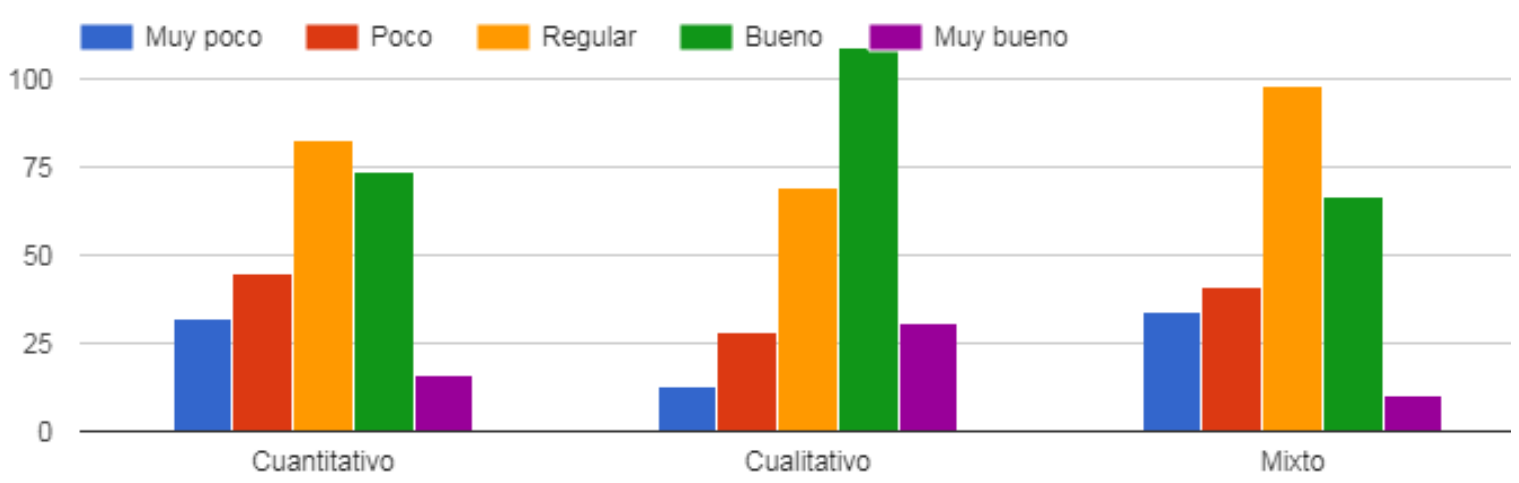

Fuente: Elaborado por los autores

El 84,4\% de los encuestados (209 personas) manifestaron haber participado en estudios e investigaciones académicas, de los cuales el $60,8 \%$, lo ha hecho en ejercicios de tipo cualitativo; $23,9 \%$ cuantitativo y $23 \%$ mixto. (Ver figura 4); en áreas como humanidades (48,1\%), Ciencias Sociales $(28,1 \%)$, Ciencias Naturales $(12,4 \%)$, Ciencias Médicas y de la salud (10\%), ingeniería y tecnología $(5,2 \%)$, ciencias agrícolas $(1,4 \%)$, educación $(1,9 \%)$ y otras áreas el porcentaje restante.

Figura 4 - Tipos de estudios o investigaciones académicas en las que ha participado

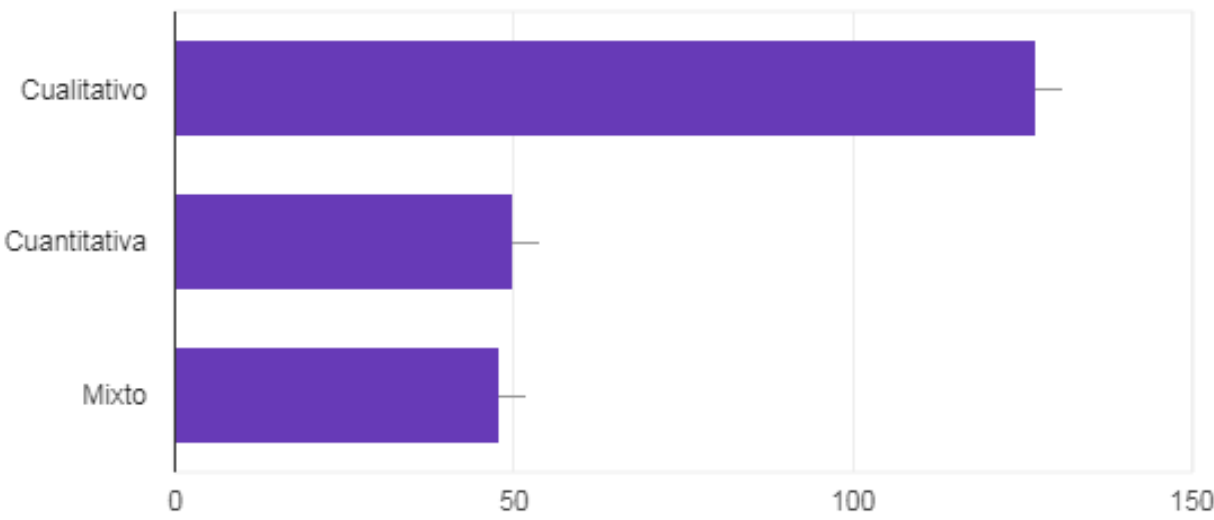

Fuente: Elaborado por los autores

También se les preguntó a las 250 personas encuestadas frente a su experiencia en la dirección de tesis, monografías y proyectos de grado, encontrando que en su gran mayoría (72,8\%) no han realizado esta labor; y quienes lo han hecho (79 personas), ha sido en el nivel de pregrado $(78,5 \%)$ y de posgrado $(31,6 \%)$ (ver Figura 5). Adicionalmente se les preguntó si 
en su actividad diaria realizan investigación, a lo que el 55,6\%, respondió de manera afirmativa (ver Figura 6).

Figura 5 - Nivel académico en el que ha participado en dirección de tesis, monografías y proyectos de grado

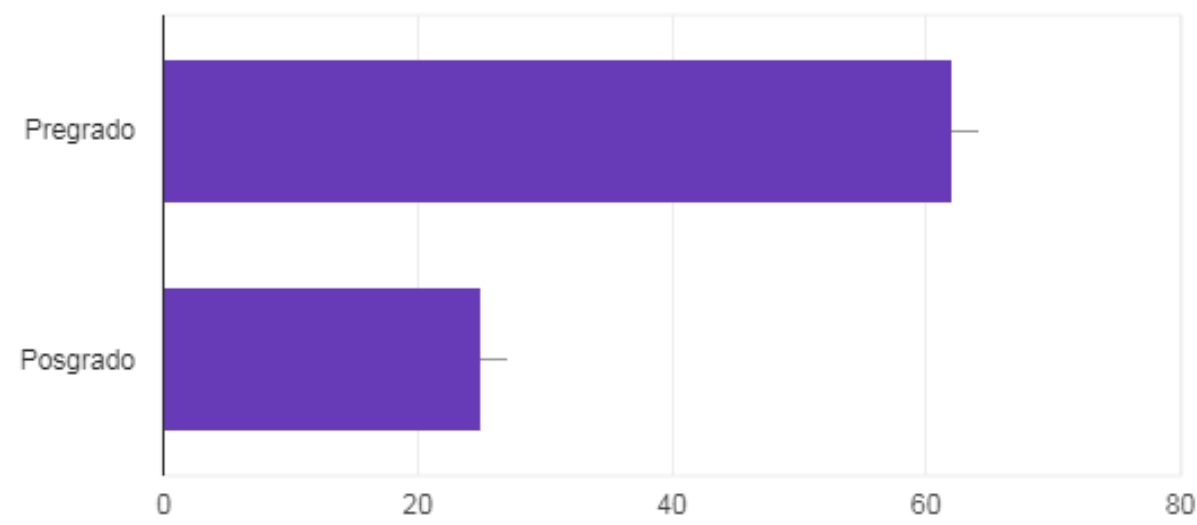

Fuente: Elaborado por los autores

Figura 6 - ¿Realiza investigación en su actividad profesional diaria?

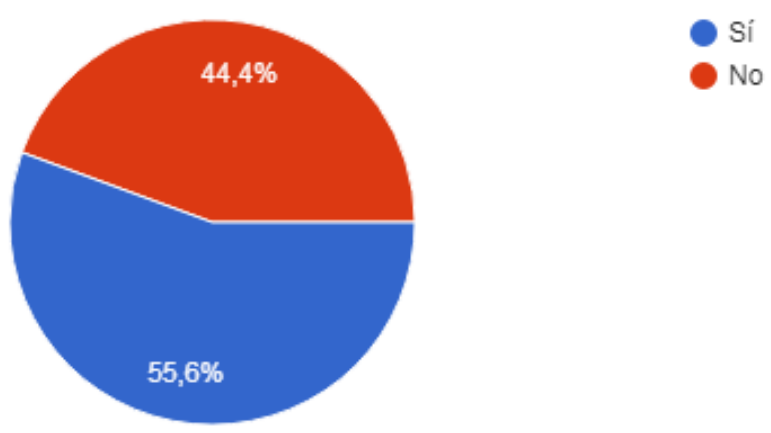

Fuente: Elaborado por los autores

- Identificar la formación y experiencia de los docentes que participan en el proceso de enseñanza y acompañamiento de la investigación. La entrevista a los docentes permitió recopilar información de 22 de ellos (54,5\% mujeres y 45,5\% hombres), que han trabajo entre 6 y 20 años en las universidades que hicieron parte de la muestra, con un contrato de tiempo completo $(81,8 \%)$. El $90,9 \%$ de los participantes tienen título de licenciado y el 71,4\% tienen título de doctorado. El 90,0\% de los docentes entrevistados trabajan en el área de Ciencias Sociales (ver gráfica 11), y el $24 \%$ ha tenido entre 20 y 21 años de experiencia como investigador. El $86.4 \%$ ha realizado trabajos de investigación en los últimos 5 años en los niveles de pregrado $(31,6 \%)$, posgrados $(57,9 \%)$ y postdoctorado $(57,9)$, con más de 10 trabajos realizados en ese periodo de tiempo $(42,1 \%)$ (ver gráfica 13), especialmente en el área de Ciencias Sociales (85\%). Además del ejercicio de investigación, los docentes han fortalecido su experiencia como investigadores a través de cursos de investigación, dirección de tesis, evaluadores de tesis, participación en eventos, entre otros. Igualmente, con participación en comités académicos $(95,5 \%)$, consejos editoriales $(68,2 \%)$, investigación formal (72,7\%), organización de eventos académicos $(81,8 \%)$. 
- Identificar las temáticas más comúnmente investigadas por los estudiantes de los posgrados en Educación y áreas afines en Ciencias Sociales y Humanas. La encuesta realizada a los estudiantes permitió definir y declarar las líneas de investigación en las que han investigado e investigan los egresados y estudiantes del programa, respectivamente. Para ellos fue necesario agrupar las diferentes temáticas en grandes líneas. En la tabla 3, es posible identificar las 6 diferentes líneas con sus respectivos temas.

Tabla 3 - Líneas de Investigación en la Maestría en Educación

\begin{tabular}{|c|c|}
\hline Líneas & Temas \\
\hline \multirow{8}{*}{ Didácticas } & Lecto Escritura \\
\hline & Matemáticas \\
\hline & Estadística \\
\hline & Ciencias Básicas y Medio Ambiente \\
\hline & Ciencias Sociales \\
\hline & Artes \\
\hline & Lenguas Extranjeras \\
\hline & Literatura \\
\hline \multirow{2}{*}{ Gestión } & Planeación Estratégica \\
\hline & Gestión y Evaluación de Proyectos Educativos \\
\hline \multirow{3}{*}{ Cultura y Ciudadanía } & Sistemas Educativos Comparados \\
\hline & Cultura y Expresión Estética \\
\hline & Formación Ciudadana \\
\hline \multirow{2}{*}{ Educación Infantil } & Políticas Públicas en Primera Infancia \\
\hline & Estrategias Pedagógicas y Didácticas En Primera Infancia \\
\hline \multirow{3}{*}{ Tecnologías en la Educación } & Incorporación De TIC \\
\hline & Innovación Educativa con TIC \\
\hline & Ambientes Virtuales \\
\hline \multirow{3}{*}{ Inclusión y Enfoque Diferencial } & $\begin{array}{l}\text { Inclusión Educativa } \\
\text { Diversidad, Enfoque Diferencial y Género }\end{array}$ \\
\hline & Deserción Escolar \\
\hline & Desarrollo Socioafectivo \\
\hline
\end{tabular}

Fuente: Documento Maestro de la Maestría en Educación UNAB (2018)

- Identificar el perfil de los estudiantes de posgrados en Educación y áreas afines en Ciencias Sociales y Humanas. Como resultado del ejercicio diagnóstico y de caracterización realizado a los estudiantes y egresados de la Maestría en Educación, se realizó una propuesta de perfil profesional y ocupacional del estudiante, atendiendo a las dos modalidades del programa profundización e investigación. La propuesta de perfil quedó dispuesta de la siguiente manera:

\section{Perfil profesional}

Modalidad de investigación: El Magíster en Educación de la UNAB será un investigador altamente especializado, con capacidad para aplicar sus conocimientos pedagógicos en la formulación y desarrollo proyectos orientados a la reflexión, análisis y generación de nuevo conocimiento que pueda aportar al contexto educativo. Será un profesional 
reflexivo y crítico con apropiación profunda sobre la fundamentación epistemológica de la educación lo que le permitirá establecer un diálogo con la comunidad científica sobre su campo de formación e investigación y contribuir así al crecimiento del cuerpo teórico del saber pedagógico en contexto.

Modalidad de profundización: El Magíster en Educación de la UNAB será un profesional sensible frente a la realidad social, cultural y educativa en los contextos local, regional y nacional en los que se desenvuelve, con capacidad para crear propuestas de intervención educativas innovadoras que ofrezcan respuesta a problemáticas vigentes, en los que lleve a la práctica el saber pedagógico construido.

\section{Perfil ocupacional}

Modalidad de investigación: El Magíster en Educación de la UNAB será un profesional sensible frente a la realidad social, cultural y educativa en los contextos local, regional y nacional en los que se desenvuelve, con capacidad para crear propuestas de intervención educativas innovadoras que ofrezcan respuesta a problemáticas vigentes, en los que lleve a la práctica el saber pedagógico construido.

Consultor en proyectos relacionados con el sistema educativo, en organizaciones públicas, privadas y ONG, del ámbito regional, nacional e internacional.

Asesor en el diseño o implementación de proyectos de investigación en educación, en los que aplica los fundamentos epistemológicos de la educación para el diagnóstico, diseño, desarrollo, implementación, evaluación, gestión, innovación.

Tutor o docente en los diferentes niveles del sistema educativo.

\section{Modalidad de profundización}

Docente o tutor en los distintos niveles del sistema educativo, en organizaciones públicas, privadas y $\mathrm{ONG}$, del ámbito regional y nacional.

Diseñador y gestor de propuestas educativas, en los que debe realizar el diagnóstico, diseño, desarrollo, implementación, evaluación, gestión e innovación para la creación y recreación de ambientes de enseñanza y aprendizaje.

Investigador en las organizaciones e instituciones educativas para la solución de problemas en contextos profesionales, organizacionales, comunitarios, sociales y globales, haciendo énfasis en las didácticas, la gestión y la cultura. 
Consultor en proyectos relacionados con el sistema educativo, en organizaciones públicas, privadas y $\mathrm{ONG}$, del ámbito regional, nacional e internacional.

\section{Conclusiones}

En este apartado se evidencian las conclusiones, discusiones y las recomendaciones a futuro relacionados con esta temática. Adicionalmente se presentarán ciertos soportes teóricos que permitirán comprobar lo expresado.

\section{Sobre el proceso de formación en investigación}

La formación en investigación requiere de un proceso planeado, previamente diseñado, que garantice que los estudiantes de posgrado logren las competencias necesarias para continuar haciendo investigación más allá del ejercicio de trabajo de grado. Para ello es necesario que al interior del programa exista un protocolo que defina momentos, actividades y entregables, así como los roles de quienes intervienen en el proceso. El protocolo de investigación propuesto en esta investigación atiende a las dos modalidades propuestas en el programa de Maestría en Educación UNAB: profundización e investigación, los cursos, tiempos y actividades necesarias para la construcción de cada capítulo. Igualmente, y producto de la recolección de información, se concluye que es necesario que el ejercicio de investigación se lleve a cabo a lo largo de todo el programa de posgrado, es decir desde el primer semestre hasta el último, con el acompañamiento de un docente a cargo de adentrar al estudiante en lo metodológico, y de un director de investigación que lo guíe en lo relacionado con el área de conocimiento y contenido de su investigación.

Lo ideal es que el estudiante investigador logre autonomía en el proceso de construcción, así como también desarrolle su pensamiento crítico, habilidades vinculadas a la búsqueda de información, análisis, síntesis, lectura y escritura.

Si bien el protocolo no puede ser el mismo para todas las universidades, si puede servir como un indicativo del proceso tanto para el estudiante, como para el docente, así como para la universidad para temas de homologación, movilidad y certificación. 


\section{Sobre los conocimientos y habilidades de ingreso de los estudiantes de posgrados}

Si bien los estudiantes al acceder a un posgrado han tenido algún tipo de acercamiento a procesos investigativos, los mismos son mínimos y no tan rigurosos como los esperados en el nivel de maestría. Por esta razón los módulos y seminarios de investigación, se convierten en aquellos a los que más temen los estudiantes, y los que más repiten a pesar de estar cuidadosamente estructurados en tiempos y actividades.

La mayoría de los estudiantes entrevistados, han tenido acercamientos a ejercicios de investigación cualitativa, con la que se sienten cómodos, pero sienten que no tienen conocimientos suficientes para emprender una investigación de corte cuantitativo o mixto. Esto aparentemente sucede, porque algunas corrientes, insisten en que el ejercicio de investigación en educación debe ser de tipo cualitativo, eliminado la posibilidad de que los estudiantes conozcan otros métodos de investigación que atiendan a sus necesidades e intereses. Por esta razón, la propuesta que se hace desde esta investigación es que los estudiantes tengan la posibilidad de recibir información sobre los diferentes tipos de investigación, y seleccionar de acuerdo con sus necesidades el que utilizarán en su trabajo de grado.

\section{Sobre la formación y experiencia de los docentes}

En general es posible decir que los docentes que tienen a cargo los procesos de investigación en las universidades tienen experiencia en el nivel de posgrados como docentes e investigadores, así como también experiencia investigativa que han obtenido gracias a la publicación de artículos en revistas indexadas, participación en ponencias, comités académicos, eventos científicos, dirección y evaluación de tesis y trabajos de grado en los diferentes niveles de formación.

Sin embargo, su experiencia en docencia en investigación está dada más por sus acciones como investigador y sus aprendizajes como estudiante de pregrado, posgrado y posdoctorado. En ese orden de ideas, se requiere un espacio de formación, que le permita al docente conocer algunos elementos relacionados con la didáctica de la investigación, así como también le ofrezca herramientas para enseñarla, facilitando su labor y garantizando que los estudiantes logren a lo largo de sus cursos, aprendizajes significativos que los motive a investigar de manera permanente sobre las necesidades del entorno, la región y el país, para generar cambio significativos en el mismo. 


\section{Sobre el currículo}

Producto de este ejercicio, es posible evidenciar que existe en la actualidad una fuerte tendencia hacia la formación posgradual en programas de Educación y específicamente en Maestría en Educación, convirtiéndose este en un programa abanderado de las universidades, en la medida en que permite adicionalmente una reflexión permanente sobre el ejercicio educativo al interior de la universidad.

Se encuentra, que las Maestrías ofertadas no solamente se soportan en los grupos de investigación de la Universidad, sino que también son estos grupos los que acompañan el proceso de investigación al interior de las mismas aportando todo su conocimiento, investigadores, líneas de trabajo, espacios de publicación, entre otros aspectos, y garantizando así el soporte científico necesario para articular académica y conceptualmente el ejercicio investigativo al interior de la maestría con la proyección institucional de la investigación.

Otro de los aspectos que llama la atención producto de este ejercicio, es el énfasis hacia los dos paradigmas de investigación: cualitativo y cuantitativo, permitiendo a los estudiantes de posgrado en el área de educación, tener muchas posibilidades, campos de acción y maneras de hacer investigaciones acordes a sus intereses y necesidades. La presencia de los dos paradigmas abre la posibilidad de incorporar innovaciones tecnológicas y educativas al ejercicio investigativo, gracias a que los "diseños cualitativos y cuantitativos se complementan, y no constituyen enfoques excluyentes en el plano de los métodos" (Escudero, 2004), obteniendo así una solución rica en diseño y no necesariamente en un único método.

A pesar de las diferencias entre un programa y otro, son más los aspectos en común que se convierten en un elemento clave para la construcción del protocolo de investigación de cara a ofrecer un ejercicio investigativo al interior de las maestrías, acorde con lo que se está haciendo a nivel nacional e internacional, y que garantice a los estudiantes el trabajo sobre competencias investigativas que permitan generar aportes, compartir conocimiento y faciliten la movilidad y continuidad entre programas.

\section{REFERENCIAS}

COLOMBIA. Ministerio de Educación Nacional (MEN). ¿Qué es la Educación Superior? 2009. Disponible en: http://www.mineducacion.gov.co/1621/article-196477.html. Acceso en: 2 feb. 2017.

COLOMBIA. Ministerio de Educación Nacional. Decreto 10001, de abril 3 de 2006. Por el cual se organiza la oferta de programas de posgrado y se dictan otras disposiciones. Disponible en: https://www.mineducacion.gov.co/1759/articles-96961_archivo_pdf.pdf. 
Acceso en: 2 feb. 2017.

COLOMBIA. Ministerio de Educación Nacional. Ley 115, de febrero 8 de 1994. Ley General de Educación. 1994. Disponible en: http://www.mineducacion.gov.co/1621/articles85906_archivo_pdf.pdf. Acceso en: 2 feb. 2017.

COLOMBIA. Ministerio de Educación Nacional. Ley 30, de diciembre 28 de 1992. Por el cual se organiza el servicio público de la Educación Superior. Disponible en:

https://www.cna.gov.co/1741/articles-186370_ley_3092.pdf. Acceso en: 2 feb. 2017.

DÁVILA, M. Tendencias recientes de los posgrados en América Latina. Colección UAI Investigación. Editorial Teseo, Universidad Abierta Interamericana, 2012.

ESCUDERO, E. Investigación Cualitativa e Investigación Cuantitativa: un Punto de Vista. Revista enfoques educacionales. v. 6, p. 11-18, 2004.

HERNÁNDEZ, R.; FERNÁNDEZ C.; BAPTISTA, M. Metodología de la investigación. ISBN: 978-607-15-0291-9. México: McGraw Hill, 2010.

OSORIO, L.; SARMIENTO, R. Modelo de enseñanza para el Diseño e Implementación de Proyectos de Investigación en Posgrados en Ciencias Sociales y Humanas. Manuscrito: Propuesta Bienal UNAB 2017-2019. 2017.

\section{Cómo referenciar este artículo}

PORRAS, R. E. S.; OSORIO VALDÉS, L. M. Framework para la enseñanza de la investigación en los posgrados de educación. Revista Ibero-Americana de Estudos em Educação, Araraquara, v. 15, n. esp. 4, p. 2653-2670, dez., 2020. e-ISSN: 1982-5587. DOI: https://doi.org/10.21723/riaee.v15iesp4.14512

Remitido el: 10/09/2019

Revisiones requeridas el: 10/01/2020

Aprobado el: 30/04/2020

Publicado el: 01/12/2020 